\title{
Identification of a new recombinant strain of echovirus 33 from children with hand, foot, and mouth disease complicated by meningitis in Yunnan, China
}

Jie Zhang ${ }^{1,2+}$, Hongbo Liu ${ }^{1,2+}$, Yilin Zhao ${ }^{1,2}$, Haihao Zhang ${ }^{1,2}$, Hao Sun ${ }^{1,2}$, Xiaoqin Huang ${ }^{1,2}$, Zhaoqing Yang ${ }^{1,2}$, Jiansheng Liu ${ }^{1,2^{*}}$ and Shaohui Ma ${ }^{1,2^{*}}$

\begin{abstract}
Background: Hand, foot, and mouth disease (HFMD) is a common childhood disease, which is usually caused by enterovirus A (EV-A) serotypes. Enterovirus A71 (EV-A71) and coxsackievirus A16 (CV-A16) are the main etiologic agents. Multiple serotypes of enterovirus B serotypes (EV-B) have been detected in outbreaks or sporadic cases of HFMD.

Results: During HFMD surveillance in Yunnan, China in 2013, two echovirus 33 (E-33) isolates were recovered in cell culture and typed by molecular methods from the cerebrospinal fluid (CSF) and feces of two sporadic cases of HFMD complicated by meningitis. Sequence analysis indicated that the study isolates, YNK35 and YNA12, formed an independent branch, and belonged to E-33 genotype $\mathrm{H}$. Recombination analysis indicated multiple recombination events in the genomic sequence of isolate YNK35. The recombination mainly occurred in the nonstructural coding region of $\mathrm{P} 2$ and $\mathrm{P} 3$, and involved intra-species recombination of species $\mathrm{B}$.

Conclusion: In this study, the complete sequences of two E-33 isolates were determined. This is the first report of severe HFMD associated with E-33 in Yunnan China, and it enriches the number of full-length genome sequences of $\mathrm{E}-33$ in the GenBank database.
\end{abstract}

Keywords: HFMD, E-33, Recombination

\section{Background}

Enteroviruses (EVs) are small, positive, single-stranded, non-enveloped RNA viruses belonging to the family Picornaviridae, which are further classified into fifteen species: enterovirus (EV)-A, EV-B, EV-C, EV-D, EV-E, EV-F, EV-G, EV-H, EV-I, EV-J, EV-K, and EV-L and rhinovirus (RV)-A, RV-B, and RV-C [1]. EVs cause a wide range of human diseases worldwide, including cutaneous, visceral, and neurological disorders. Among these diseases, hand, foot, and mouth disease (HFMD) is a common childhood disorder which typically presents as a brief, febrile illness

\footnotetext{
*Correspondence: ljsh3300@163.com; shaohuima70@hotmail.com; shaohuima70@126.com

${ }^{\dagger}$ Jie Zhang and Hongbo Liu contributed equally to this work.

'Institute of Medical Biology, Chinese Academy of Medical Sciences and Peking Union Medical College, Kunming 650118, People's Republic of China Full list of author information is available at the end of the article
}

characterized by the association of oral ulcerations (enanthema) and vesicular rash (exanthema) on the palms, soles, and buttocks [2]. EV-A71 and CV-A16 are the major etiological agents of HFMD [3, 4]. Serotypes of EV-B (Es) have been mainly related to herpangina and seasonal outbreaks of mild viral meningitis, but have recently been associated with epidemics of HFMD. Echovirus (E) belongs to species EV-B. And multiple E serotypes are detected in sporadic cases or outbreaks of HFMD, and frequently co-circulated with EV-A71 and CV-A16 in large epidemics, including E-3, E-4, E-5, E-6, E-7, E-9, E-11, E-16, E-17, E-18, E-24, E-25, and E-30 [5-22].

In 1959, echovirus 33 (E-33) was isolated, and was identified as the last Enteric Cytopathic Human Orphan (ECHO) virus belonging to the species enterovirus $B$ in 1963 [23]. Since then, E-33 has been detected in several outbreaks and 
sporadic cases around the world, associated with rashes, diarrhea, fever, aseptic meningitis, encephalitis, acute flaccid paralysis (AFP), and influenza-like illness [24-29]. The prototype strain (Toluca-3) of E-33 was isolated in 1959 [23]. To date, there are only two full-length genome sequences in the GenBank database, including the prototype strain and another E-33 strain isolated in 2014, and clinical information on E-33 has not been reported. During HFMD surveillance, two E-33 viruses were isolated, from stool and CSF, and identified in two sporadic cases of HFMD complicated by meningitis. To our knowledge, this was the first study to report severe HFMD caused by E-33 in Yunnan China.

\section{Results}

\section{Primary characterization of the virus isolates}

The isolates YNK35/CHN/2013 and YNA12/CHN/2013 were recovered from human embryonic lung diploid fibroblast (KMB17), human rhabdomyosarcoma (RD), and human lung cancer (A549) cell lines. YNK35 and YNA12 were isolated from CSF and stool samples from two sporadic cases of severe HFMD complicated by meningitis. Capsid protein VP1 sequencing and molecular typing using BLAST [30] revealed that these two study isolates were E-33.

When compared with the entire VP1 coding sequences of different prototype EVs retrieved from GenBank, YNK35 and YNA12 were found to have high homology with E-33, with $78.2-78.3 \%$ identity to the E-33 prototype strain, Toluca- 3 , but $<70 \%$ identity to the VP1 sequences of all other enterovirus serotypes. It is proposed that YNK35 and YNA12 isolates belong to E-33, according to the enterovirus type demarcation criterion (that strains with $>75 \%$ nucleotide or $>88 \%$ amino acid homology of VP1 sequences belong to the same serotype).

\section{VP1 sequence analysis}

Phylogenetic trees were constructed by aligning the complete VP1 sequences of the two E-33 study isolates with other E-33 sequences retrieved from GenBank. In order to study the genetic diversity and possible relationships of the E-33 strains, 31 E-33 sequences available in GenBank were collected from different country or different date. The phylogenetic tree revealed that global E-33 strains could be grouped into eight major distinct phylogenetic clusters, corresponding to A-H (Fig. 1). Prototype Toluca-3 was the only strain in cluster A. The topology of the phylogenetic tree indicated that the study Yunnan isolates YNK35 and YNA12 isolated in 2013 belonged to genotype $\mathrm{H}$ and formed an independent branch. However, one Yunnan isolate (316-YN-CHN-2015JK) isolated in 2015 belonged to genotype C. Most Chinese E-33 isolates, ILIHuN13-1, ILIHuN13-6, ILIHuN13-9, 61-YN-CHN-2014JK, 64-YN-CHN-2014JK, 65-YN-CHN-2014JK, and HB92 clustered as genotype $\mathrm{G}$.

Homology comparisons of the complete VP1 nucleotide and amino acid sequences were performed among the E-33 genotypes (Additional file 1: Table S1). The average VP1 nucleotide sequence divergence within the E-33 genotypes was $17.5 \%$ (12.1-22.9\%). In particular, for genotype $\mathrm{H}$, which had nucleotide divergence of $17.75 \%(17.1-18.4 \%)$ and $17.0 \%(16.3-17.7 \%)$, respectively. When compared with genotypes $\mathrm{G}$ and $\mathrm{C}$, all sequences were above the mean $14.95 \%$ cutoff divergence value assigned for EV-A71 subgenotyping [31]. YNK35 and YNA12 had 81.6-82.6\% (98.6-99.3\%) nucleotide (amino acid) sequence similarity with other Chinese E-33 isolates with complete VP1 sequences.

\section{Complete genome analysis}

Isolates YNK35 and YNA12 were found to be closely related, having $99.0 \%$ nucleotide (98.2\% amino acid) identity for the entire genome, so the complete genomic sequence of isolate YNK35 was characterized as representative in the following analysis. The complete genome of isolate YNK35 consisted of 7392 nucleotides, including a 6552-nucleotide open reading frame (ORF) encoding a potential polyprotein precursor of 2183 amino acids, a 5 '-untranslated region (UTR) of 743 nucleotides and a 3'-UTR of 97 nucleotides. In comparison with the prototype strain Toluca-3, it had $79.9 \%$ similarity and $95.7 \%$ similarity in the complete genome and deduced amino acid sequences of the potential polyprotein precursor, respectively. No nucleotide deletions or insertions were found in alignment of isolate YNK35 and the prototype strain. In addition, isolate YNK35 had 81\% nucleotide and 96.4\% amino acid similarity, respectively, with the only Chinese E-33 isolate with a complete genome deposited in the GenBank, which was named HB92 but was not typed as E-33 by the authors. Table 1 shows a comprehensive comparison of different genomic regions of the nucleotide sequence and the deduced amino acid sequence of isolate YNK35 with the E-33 prototype strain and HB92 isolate.

\section{Phylogenetic analysis based on $P 1, P 2, P 3$ coding regions}

Construction of phylogenetic trees by aligning the sequences of the coding regions $P 1, P 2$, and $P 3$ of YNK35 and YNA12 with 383 EV-B strains available in the GenBank database are shown in Fig. 2. In the $P 1$ capsid coding region, YNK35 and YNA12 formed a lineage, with a bootstrap value of $100 \%$. Meanwhile, the two study isolates formed a cluster with all other E-33 strains, such as Toluca-3, HB92 and PMKA0914/1174 (which were isolated from undiagnosed respiratory specimens in Thailand [32]), correspondent with the preliminary molecular typing results based on the complete VP1 sequences.

The phylogeny of the non-structural protein coding regions displayed different results; the isolates YNK35 and YNA12 were interspersed with other EV isolates. In the P2 coding region, YNK35 and YNA12 were closely related to CV-B3, EV-86, and E-25. In the P3 coding region, YNK35 


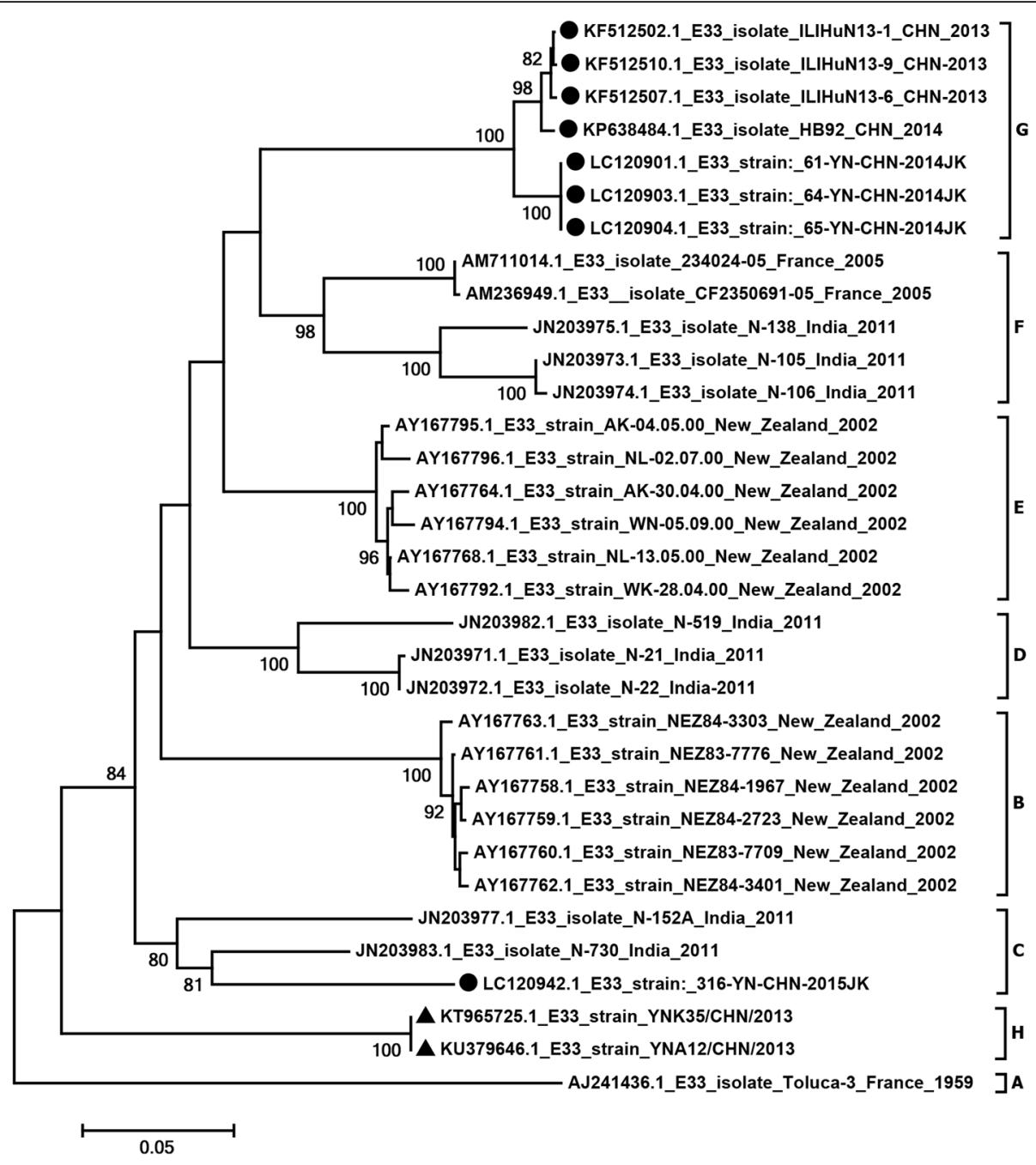

Fig. 1 Phylogeny of the complete VP1 sequences of 98 E-33 strains. The MEGA 6.06 program was utilized to analyze the relationships between the two Yunnan E-33 isolates and the other 96 E-33 isolates available in the GenBank database. Numbers at the nodes represent bootstrap values supported for that node (1000 bootstrap replicates). Only high bootstrap values (>75\%) are shown. The scale bars indicate the genetic distance. $\boldsymbol{\Delta}$ strains were isolated in this study, and $\bullet$ strains were collected from other provinces in China. The Complete trees are available as Additional file 1: Figure S1

and YNA12 were very similar to E-4, EV-B106, and E-6. These results suggested that one or more putative recombination events had probably occurred between the study isolates and other EV serotypes.

\section{Recombination analysis}

To confirm the recombination events occurring between the Yunnan E-33 isolates and other EV-B serotypes, a similarity plot was produced and bootscanning analyses were performed (Fig. 3). The similarity plot and bootscanning analyses both suggested multiple recombination events in the genomic sequence of isolates YNK35 and YNA12. In the P1 and $2 C$ coding regions, isolate YNK35 had the highest identity with the E-33 isolate HB92. However, in the $5^{\prime}$-UTR, isolate YNK35 was most homologous with the CV-B3 strain
GZ803. For the $2 A$ coding region, it was most similar to the E-20 strain KM-EV20-2010. In the 3B coding region, YNK35 showed highest identity with the E-3 strain HNWY-01. In addition, YNK35 showed highest identity with the E-4 strain AUS250G and E-6 strain Echo6/Henan/116/2008, in the partial $3 C$ coding region and the $3 D$ coding region, respectively.

\section{Discussion}

In this study, YNK35 was isolated from CSF, and YNA12 was isolated feces. The patients displayed a similar clinical presentation: hand, foot, and mouth disease complicated by aseptic meningitis. Both isolates produced typical enterovirus-like cytopathic effect (CPE) on KMB17, RD, and A549 cell lines; enterovirus molecular serotyping confirmed the presence of E-33. The E-33 isolate YNK35 was 
Table 1 Nucleotide and amino acid identities between Toluca-3/HB92 and YNK35/CHN/2013 in different genomic regions

\begin{tabular}{lllll}
\hline Genomic region & \multicolumn{2}{l}{ Toluca-3 } & & HB92 \\
\cline { 2 - 2 } \cline { 5 - 6 } \% Nucleotide identity & \% Amino acid identity & & \% Nucleotide identity & Amino acid identity \\
\hline 5'UTR & 83.4 & & 85.3 & 98.6 \\
VP4 & 79.0 & 97.1 & 79.2 & 94.9 \\
VP2 & 77.9 & 90.8 & 81.0 & 97.1 \\
VP3 & 78.4 & 95.4 & 81.8 & 99.3 \\
VP1 & 78.2 & 96.4 & 82.4 & 94.7 \\
2A & 77.1 & 92.0 & 81.1 & 93.8 \\
2B & 76.1 & 95.9 & 78.2 & 98.2 \\
2C & 81.0 & 99.4 & 80.2 & 95.5 \\
3A & 81.3 & 93.3 & 80.9 & 100 \\
3B & 77.0 & 95.0 & 80.3 & 97.3 \\
3C & 80.9 & 98.9 & 77.4 & 96.3 \\
3D & 81.3 & 96.3 & 80.7 & 96.4 \\
3'UTR & 84.4 & & 84.5 & \\
Complete genome & 79.9 & 95.7 & 81.0 & \\
\hline
\end{tabular}

isolated from CSF, which is the best specimen for the conclusive identification of the pathogenic agent in a patient with meningitis. The Chinese E-33 isolates, ILIHuN13-1, ILIHuN13-6, and ILIHuN13-9, were responsible for a large outbreak of influenza-like illness (ILI) in Hunan Province, China in 2013 [25]. Other isolates, $61-\mathrm{YN}-\mathrm{CHN}-2014 \mathrm{JK}, \quad 64-\mathrm{YN}-\mathrm{CHN}-2014 \mathrm{JK}$, $65-\mathrm{YN}-\mathrm{CHN}-2014 \mathrm{JK}$ and $316-\mathrm{YN}-\mathrm{CHN}-2014 \mathrm{JK}$ were detected in healthy children. And no clinical information, molecular epidemiology, or disease correlation of the Chinese isolate HB92 has been reported. Therefore, the etiological characteristics of E-33 in China still need further study.

Sequence analysis indicated that the study isolates YNK35 and YNA12 could be regarded as a separate genotype in the near future, whereas the contemporary Chinese E-33 isolates, ILIHuN13 and HB92 were clustered as genotype G. This implies that there were at least two E-33 genotypes circulating in mainland China in that era. In addition, previous research confirmed that partial sequences of sporadic E-33 strains were identified in feces from healthy children in Yunnan, China [33]. Therefore, before these pathogenic E-33 strains appeared, they may have formed a new genomic lineage that had been circulating "silently" for some years within the same geographical area.

Recombination drives the evolution of EVs and usually occurs in the non-structural regions of EV-B [34-38]. The recombination analysis suggested that multiple recombination events had occurred in the genomic sequence of isolates YNK35 and YNA12. In this study, recombination events were detected between isolate YNK35 (or YNA12) and other EV-B strains, such as EV-86 strains, CV-B3 strains, E-4 strains, and the

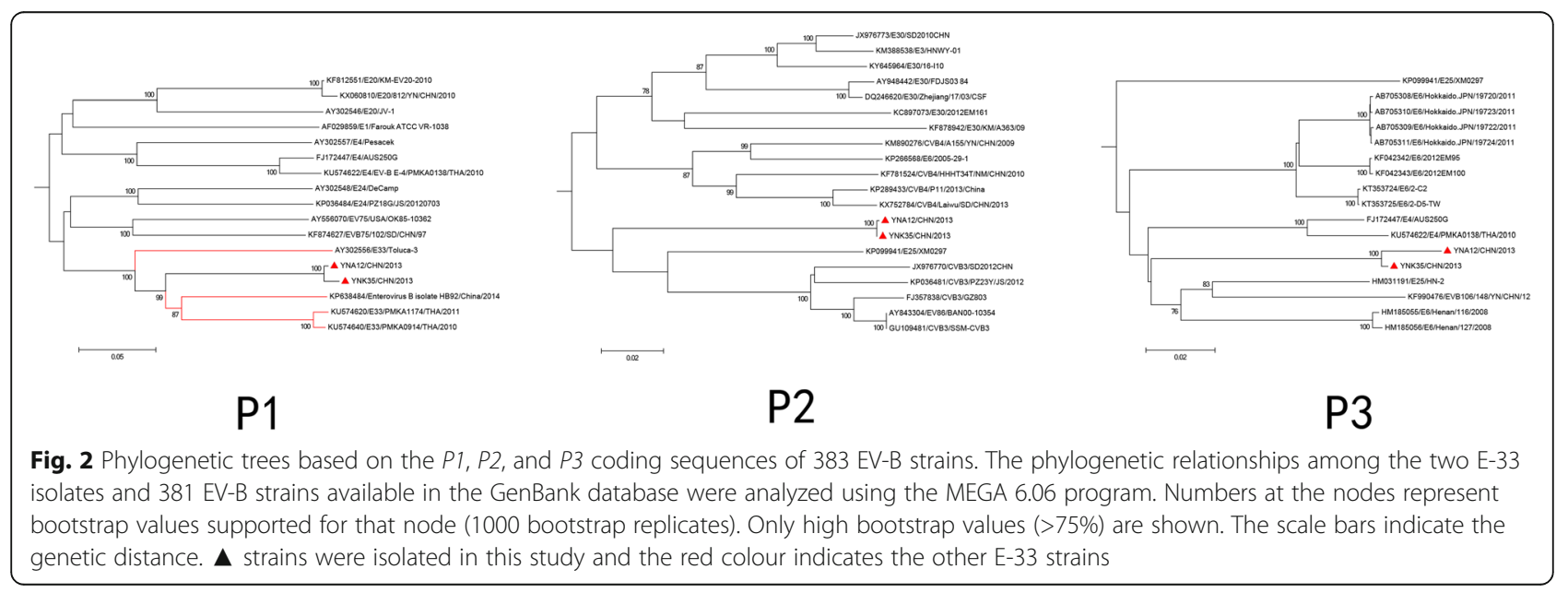



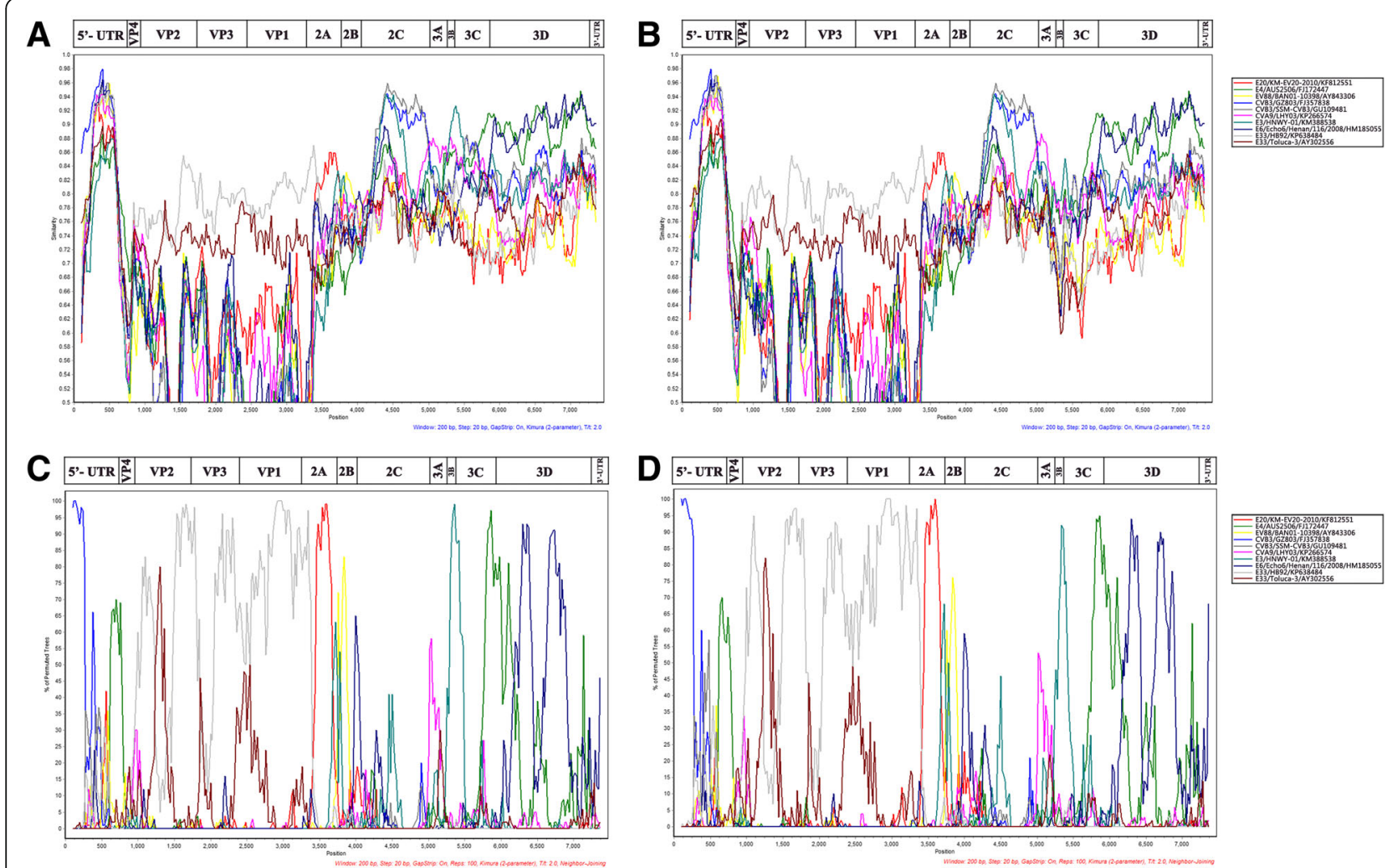

Fig. 3 Similarity plot and bootscanning analyses of the complete genome of the two Yunnan E-33 strains. Similarity plot (a/b) and bootscanning analysis (c/d) of complete EV-B genomes use a sliding window of 200 nucleotides moving in steps of 20 nucleotides. The genome of isolate YNK35/CHN/2013 (a/c), YNA12/CHN/2013 (b/d) serves as a query sequence, independently

EV-B106 strain. The recombination had mainly occurred in the non-structural coding region of $P 2$ and $P 3$ among EV-B strains, representing the intra-species recombination. The recombination donors were usually contemporary predominant circulating EV-B; it is worth noting that they were isolated from different geographical locations around the world. Whether or how these recombinations may have contributed to the emergence of lineages with modified pathogenic properties (such as severe HFMD) also needs to be investigated further.

\section{Conclusion}

In summary, we have reported the entire genome sequences of two E-33 isolates isolated during HFMD surveillance in Yunnan, China in 2013. Analysis of the nucleotide sequences revealed that these two Yunnan isolates have high genetic diversity when compared with the E-33 prototype strain, showing intertypic recombination in the non-structural protein encoding region, and genetic exchanges with other EV-B strains. Until now, only two E-33 isolates with complete genome sequences have been deposited in GenBank. The study enriched the number of full-length genome sequences of E-33 in the GenBank database, as well as contributing to the molecular epidemiology of E-33 in China, which is of great importance when evaluating the association between E-33 and E-33-related diseases.

\section{Methods}

Samples and virus isolation

During HFMD surveillance (China Information System for Disease Control and Prevention) in 2013, two patients with HFMD (with rashes on hands, feet, knees, and buttocks), a 1-year and 6-month-old girl and a 1-year and 2-month-old boy, were admitted to a pediatric hospital in Kunming (China). Both children manifested nonspecific fever of 38.9$39.3^{\circ} \mathrm{C}$ and meningeal signs of a sore or stiff neck. CSF and stool specimens were collected from the two patients. To make a thorough attempt to isolate poliovirus, RD, A549, and KMB17 cell lines were used, in accordance with standard protocol [39]. All positive isolates (cell cultures with CPE appearance) were stored at $-80^{\circ} \mathrm{C}$ until use.

\section{Viral RNA extraction, reverse transcription-polymerase chain reaction (RT-PCR), sequencing and typing}

Nucleic acid was extracted from cell culture supernatants with an AxyPrep Body Fluid Viral DNA/RNA Miniprep Kit (Axygen, Union City, CA, USA), which was used in accordance with the manufacturer's instructions. The RT-PCR 
reactions were performed with a PrimeScript ${ }^{\mathrm{mi}}$ One Step RT-PCR Kit Ver.2 (Takara, Dalian, China) to amplify the partial VP1 gene by using VP1 gene-specific primer pairs 222 and 224 [40]. These sequences of partial VP1 were uploaded into GenBank BLAST (http://www.ncbi.nlm.nih.gov/BLAST/) for direct identification of enterovirus serotypes. The primers (Additional file 1: Table S2) used for amplification of the complete genomes were based on the prototype strain, and were designed using a "primer-walking" strategy [41]. An ABI 3730XL automatic sequencer (Applied Biosystems, Foster City, CA, USA) was used to sequence the resulting DNA templates at Tsingke Biological Technology Co., Ltd., Beijing.

\section{Sequence analysis and recombination analysis}

Phylogenetic trees were constructed by Molecular Evolutionary Genetic Analysis (MEGA) version 6.06 software, using the neighbour-joining algorithms with 1000 bootstrap replicates. Simplot software version 3.5.1 was used to analyze the plot of nucleotide similarities, with a sliding window of 200 nucleotides moving in 20-nucleotide steps [41]. Pairwise alignments of the sequences were carried out via Geneious Basic 5.6.5 software [41].

\section{The GenBank accession number of the nucleotide sequences}

The complete genome sequences of the E-33 isolates YNK35/CHN/2013 and YNA12/CHN/2013 identified in this study are available under the GenBank accession numbers KT965725 and KU379646.

\section{Additional file}

Additional file 1: Supplementary Information. (DOC $63250 \mathrm{~kb}$ )

\section{Abbreviations}

5'UTR: 5'-untranslated region; CPE: cytopathic effect; E-33: Echovirus 33; Es: Echoviruses; EVs: Enteroviruses

\section{Acknowledgements}

Not applicable.

\section{Funding}

This work was supported by the Basic Research Projects of Yunnan Province, China (2017FA006) and Chinese Academy of Medical Sciences Initiative for Innovative Medicine (2016-|2M-3-026).

\section{Availability of data and materials}

The data presented in this manuscript are therein. Those not presented have been stored in GenBank.

\section{Authors' contributions}

$J Z$, JL, and SM conceived the study and drafted the paper, JZ, HL, HZ, and $Y Z$ performed the experiments. $H S, X H$, and $Z Y$, helped to interpret results and contributed to the writing. All authors reviewed the manuscript. All authors read and approved the final manuscript.

\section{Ethics approval and consent to participate}

All participants gave written informed consent. The protocol was approved by the Institutional Review Boards of the Institute of Medical Biology,
Chinese Academy of Medical Sciences \& Peking Union Medical College and adhered to the Declaration of Helsinki.

\section{Consent for publication \\ Not applicable.}

\section{Competing interests}

The authors declare that they have no competing interests.

\section{Publisher's Note}

Springer Nature remains neutral with regard to jurisdictional claims in published maps and institutional affiliations.

\section{Author details}

${ }^{1}$ Institute of Medical Biology, Chinese Academy of Medical Sciences and Peking Union Medical College, Kunming 650118, People's Republic of China. ${ }^{2}$ Yunnan Key Laboratory of Vaccine Research Development on Severe Infectious Disease, Kunming 650118, People's Republic of China.

Received: 19 December 2018 Accepted: 15 April 2019

Published online: 08 May 2019

\section{References}

1. Adams MJ, King AM, Carstens EB. Ratification vote on taxonomic proposals to the international committee on taxonomy of viruses (2013). Arch Virol. 2013:158:2023-30. https://doi.org/10.1007/s00705-013-1688-5.

2. Modlin JF. Coxsackieviruses, Echoviruses and newer enteroviruses. In: Mandell $\mathrm{GL}$, Bennet JE, Dolin R, editors. Principles and practice of infectious diseases. 4th ed. London, UK: Elsevier Churchill Livingstone; 2000. p. 1904-19.

3. Podin Y, et al. Sentinel surveillance for human Enterovirus 71 in Sarawak, Malaysia: lessons from the first 7 years. BMC Public Health. 2006;6:180. https://doi.org/10.1186/1471-2458-6-180.

4. Van Tu P, et al. Epidemiologic and Virologic investigation of hand, foot, and mouth disease, southern Vietnam, 2005. Emerg Infect Dis. 2007;13:1733-41. https://doi.org/10.3201/eid1311.070632.

5. Russo DH, Luchs A, Machado BC, Carmona RC, Timenetsky MC. Echovirus 4 associated to hand, foot and mouth disease. Rev Inst Med Trop Sao Paulo. 2006:48:197-9.

6. Han JF, et al. Echovirus 30 in EV71-associated hand, foot and mouth disease outbreak, Guangxi, China. J Clin Virol. 2011;50:348-9. https:/doi.org/10.1016/j.jcv.2011.01.005.

7. Bian, L. L. et al. Complete genome sequence analysis of echovirus 24 associated with hand-foot-and-mouth disease in China in 2012. Genome Announc 3, e01456e1414-e1456, doi: https://doi.org/10.1128/genomeA.01456-14 (2015).

8. Hongyan G, et al. Hand, Foot and Mouth Disease Caused by Coxsackievirus A6, Beijing, 2013. Pediatr Infect Dis J. 2014;33:1302-3. https://doi.org/10. 1097/INF.0000000000000467.

9. Han JF, et al. Hand, Foot, and Mouth Disease Outbreak Caused by Coxsackievirus A6, China, 2013. J Inf Secur. 2014;69:303-5. https://doi.org/10. 1016/j.jinf.2014.03.015

10. Guan HY, et al. Pathogenic Spectrum of enteroviruses associated with hand, foot and mouth disease by a GeXP-based multiplex reverse transcriptionPCR assay in Jinan, China, 2009-2012. Bing Du Xue Bao. 2014;30:567-71.

11. Zhang $X$, et al. Prevalence of enteroviruses in children with and without hand, foot, and mouth disease in China. BMC Infect Dis. 2013;13:606. https://doi.org/10.1186/1471-2334-13-606.

12. Du J, et al. Changing Aetiology of hand, foot and mouth disease in Linyi, China, 2009-2011. Clin Microbiol Infect. 2014;20:047-9. https://doi.org/10.1111/1469-0691.12301.

13. Tao Z, et al. Molecular epidemiology of human Enterovirus associated with aseptic meningitis in Shandong Province, China, 2006-2012. PLoS One. 2014 9:e89766. https://doi.org/10.1371/journal.pone.0089766.

14. Lee MS, et al. Challenges to licensure of Enterovirus 71 vaccines. PLoS Negl Trop Dis. 2012;6:e1737. https://doi.org/10.1371/journal.pntd.0001737.

15. Gopalkrishna V, Patil PR, Patil GP, Chitambar SD. Circulation of multiple Enterovirus serotypes causing hand, foot and mouth disease in India. J Med Microbiol. 2012;61:420-5. https://doi.org/10.1099/jmm.0.036400-0.

16. Chen KT, Chang HL, Wang ST, Cheng YT, Yang JY. Epidemiologic Features of Hand-Foot-Mouth Disease and Herpangina Caused by Enterovirus 71 in Taiwan, 1998-2005. Pediatrics. 2007;120:e244-52. https://doi.org/10.1542/peds.2006-3331.

17. Lo SH, et al. Clinical and Epidemiologic Features of Coxsackievirus A6 Infection in Children in Northern Taiwan Between 2004 and 2009. J 
Microbiol Immunol Infect. 2011;44:252-7. https://doi.org/10.1016/j.jmii. 2011.01.031.

18. Puenpa J, et al. Prevalence and characterization of Enterovirus infections among pediatric patients with hand foot mouth disease, Herpangina and influenza like illness in Thailand, 2012. PLoS One. 2014; 9:e98888. https://doi.org/10.1371/journal.pone.0098888.

19. Mirand A, et al. Outbreak of hand, foot and mouth disease/Herpangina associated with Coxsackievirus A6 and A10 infections in 2010, France: a large citywide, prospective observational study. Clin Microbiol Infect. 2012; 18:E1 10-8. https://doi.org/10.1111/j.1469-0691.2012.03789.x.

20. Kapusinszky B, Szomor KN, Farkas A, Takacs M, Berencsi G. Detection of Non-Polio Enteroviruses in Hungary 2000-2008 and Molecular Epidemiology of Enterovirus 71, Coxsackievirus A16, and Echovirus 30. Virus Genes. 2010; 40:163-73. https://doi.org/10.1007/s11262-009-0440-4.

21. Bracho MA, Gonzalez-Candelas F, Valero A, Cordoba J, Salazar A. Enterovirus coinfections and Onychomadesis after hand, foot, and mouth disease, Spain, 2008. Emerg Infect Dis. 2011;17:2223-31. https//doi.org/10.3201/eid1712.110395.

22. Davia $J \mathrm{~L}$, et al. Onychomadesis outbreak in Valencia, Spain associated with hand, foot, and mouth disease caused by enteroviruses. Pediatr Dermatol. 2011;28:1-5. https://doi.org/10.1111/j.1525-1470.2010.01161.x.

23. ROSEN L, KERN J. Toluca-3, a newly recognized Enterovirus. Proc Soc Exp Biol Med. 1965;118:389-91.

24. Huang QS, et al. An echovirus type 33 winter outbreak in New Zealand. Clin Infect Dis. 2003;37:650-7. https://doi.org/10.1086/376915.

25. Tan X, et al. An outbreak of echovirus 33 in schools in China in 2013. Arch Virol. 2014;159:2233-41. https://doi.org/10.1007/s00705-014-2059-6.

26. Papadakis G, Chibo D, Druce J, Catton M, Birch C. Detection and genotyping of enteroviruses in cerebrospinal fluid in patients in Victoria, Australia, 2007-2013. J Med Virol. 2014;86:1609-13. https://doi.org/10.1002/jmv.23885.

27. Grimwood K, et al. Acute flaccid paralysis from echovirus type 33 infection. J Clin Microbiol. 2003:41:2230-2.

28. Sato $K$, et al. A new-born baby outbreak of echovirus type 33 infection. J Inf Secur. 1998;37:123-6.

29. Kelen AE, Lesiak JM, Labzoffsky NA. Occurrence of echovirus 33 infections in Ontario. Can Med Assoc J. 1968;98:985-7.

30. Kroneman A, et al. An automated genotyping tool for enteroviruses and noroviruses. J Clin Virol. 2011;51:121-5. https://doi.org/10.1016/j.jcv.2011.03.006.

31. Chan YF, Sam IC, AbuBakar S. Phylogenetic designation of Enterovirus 71 genotypes and subgenotypes using complete genome sequences. Infect Genet Evol. 2010;10:404-12. https://doi.org/10.1016/..meegid.2009.05.010.

32. Zhou Y, et al. Metagenomics study of viral pathogens in undiagnosed respiratory specimens and identification of human enteroviruses at a Thailand hospital. Am J Trop Med Hyg. 2016;95:663-9. https:/doi.org/10.4269/ajtmh. 16-0062.

33. Tian BJ, Ding ZR, Chen SM. Molecular test of enteroviruses for healthy children in Yunnan Province and the border areas with Myanmar, 2012. Chinese Journal of Vaccines \& Immunization. 2013;19:419-23.

34. Lukashev AN, et al. Recombination in circulating human Enterovirus B: independent evolution of structural and non-structural genome regions. J Gen Virol. 2005;86:3281-90. https://doi.org/10.1099/vir.0.81264-0.

35. Song $Y$, et al. Phylogenetic characterizations of highly mutated EV-B106 recombinants showing extensive genetic exchanges with other EV-B in Xinjiang, China. Sci Rep. 2017;7:43080. https://doi.org/10.1038/srep43080.

36. Zhang J, et al. Molecular characterization of a new human Coxsackievirus B2 associated with severe hand-foot-mouth disease in Yunnan Province of China in 2012. Arch Virol. 2017;162:307-11. https://doi.org/10.1007/s00705-016-3075-5.

37. Zheng $\mathrm{H}$, et al. Isolation and characterization of a highly mutated Chinese isolate of Enterovirus B84 from a patient with acute flaccid paralysis. Sci Rep. 2016;6:31059. https://doi.org/10.1038/srep31059.

38. Zhang $\mathrm{H}$, et al. Molecular characterization of two novel echovirus 18 recombinants associated with hand-foot-mouth disease. Sci Rep. 2017;7: 8448. https://doi.org/10.1038/s41598-017-09038-y.

39. Prim N, et al. Combining cell lines to optimize isolation of human Enterovirus from clinical specimens: report of 25 years of experience. J Med Virol. 2013;85:116-20. https://doi.org/10.1002/jmv.23426.

40. Nix WA, Oberste MS, Pallansch MA. Sensitive, Seminested PCR amplification of VP1 sequences for direct identification of all Enterovirus serotypes from original clinical specimens. J Clin Microbiol. 2006;44: 2698-704. https://doi.org/10.1128/JCM.00542-06.

41. Liu, J. et al. Complete Genome Sequence Analysis of Two Human Coxsackievirus A9 Strains Isolated in Yunnan, China, in 2009. Virus Genes. 50, 358-364, doi:https://doi.org/10.1007/s11262-015-1180-2 (2015).

Ready to submit your research? Choose BMC and benefit from:

- fast, convenient online submission

- thorough peer review by experienced researchers in your field

- rapid publication on acceptance

- support for research data, including large and complex data types

- gold Open Access which fosters wider collaboration and increased citations

- maximum visibility for your research: over $100 \mathrm{M}$ website views per year

At BMC, research is always in progress.

Learn more biomedcentral.com/submissions 